QUARTERLY OF APPLIED MATHEMATICS

VOLUME LXX, NUMBER 4

DECEMBER 2012, PAGES 743-758

S 0033-569X(2012)01274-4

Article electronically published on June 22, 2012

\title{
EXCHANGE OF STABILITIES IN COUETTE FLOW BETWEEN CYLINDERS WITH NAVIER-SLIP CONDITIONS
}

\author{
BY \\ ISOM H. HERRON (Department of Mathematical Sciences, Rensselaer Polytechnic Institute, Troy, \\ New York) \\ AND \\ PABLO U. SUÁREZ (Department of Mathematical Sciences, Delaware State University, Dover, \\ Delaware)
}

\begin{abstract}
Viscous Couette flow is derived for flow between two infinitely long concentric rotating cylinders with Navier slip on both. Its axisymmetric linear stability is studied within a regime that would be hydrodynamically stable according to Rayleigh's criterion: opposing gradients of angular velocity and specific angular momentum, based on the rotation rates and radii of the cylinders. Stability conditions are analyzed, by methods based on those of Synge and Chandrasekhar. For sufficiently small slip length on the outer cylinder no instability occurs with arbitrary slip length on the inner cylinder. As a corollary, slip on the inner cylinder is shown to be stabilizing, with no slip on the outer cylinder. Two slip configurations are investigated numerically, first with slip only on the outer cylinder, then second with equal slip on both cylinders. It is found that instability does occur (for large outer slip length), and the principle of exchange of stabilities emerges. The instability disappears for sufficiently large slip length in the second case; Rayleigh's criterion provides an explanation for these phenomena.
\end{abstract}

1. Introduction. In many areas of science, some new developments are best understood in the light of older ones with a modern lens, when it was thought that they were fully explored. Viscometry accounts for much of the early interest in flows between rotating cylinders, as Joseph [9] points out:"By integrating the shear stresses of a fluid in Couette flow with cylindrical walls we find a linear relation between torque and viscosity... But this linear relation holds only so long as the flow is stable." Explanations, due to Rayleigh, originally began with a stability theory of inviscid revolving fluids. When the instability of flow between rotating cylinders is studied in the viscous regime, many of the features are typical of a large class of problems. This was carried out by

Received December 22, 2010.

2010 Mathematics Subject Classification. Primary 76E07; Secondary 76U05, 76A02.

This paper is in final form and no version of it will be submitted for publication elsewhere.

E-mail address: herroi@rpi.edu

E-mail address: psuarez@dsu.edu

(C)2012 Brown University Reverts to public domain 28 years from publication 
G. I. Taylor in 1923. By an ingenious blend of experiment and theory, he was able to conclude that when the cylinders rotate in the same direction, an instability, observed in the form of vortices, could be described quite accurately mathematically. A companion result, one generalizing Rayleigh's criterion for inviscid flows, was later derived by Synge [14] (see also [2] and [7]): Viscous stability holds if

$$
\Omega_{1}>\Omega_{2} \text { and } \Omega_{2} r_{2}^{2}>\Omega_{1} r_{1}^{2},
$$

where $\Omega_{1}$ and $r_{1}$ are the angular velocity and radius respectively of the inner cylinder, and $\Omega_{2}$ and $r_{2}$ are the corresponding quantities for the outer cylinder.

In recent years several of the classical fluid problems of this type have been recast to model flows on a nanoscale or microscale ([1], 4]), so the Navier-slip conditions become relevant in certain applications related to hemodynamics and high-altitude flows as well. It has long been recognized that stress-free conditions, the counterpart of infinite slip length, may apply at a fluid-gas interface ([16, [12]). Given the recent interest in microscale flows, it is timely to investigate the classical stability problems to address the effect of slip conditions and determine how adding slip would affect an existing instability. Apace with these ideas have been others of adding coatings to reduce drag at walls. Applications to Couette flow have provided some interesting results in which slip was allowed on the inner cylinder ([17], [18]); in the first paper, $\Omega_{1}=0$ and no instability was noted, while in the second one, $\Omega_{2}=0$, and instability was studied.

The first objective of the current work, explored in the next section, is to derive the basic flow which results from applying the Navier-slip conditions on both cylinders; i.e., the velocity at a surface is proportional to the tangential viscous stress. Owing to the fact that the surfaces are curved, cylindrical coordinates are appropriate. The equations of motion are unchanged, but due to the unusual boundary conditions the Couette flow, though having the same functional appearance, behaves differently from the no-slip case. There are slip lengths $\ell_{1}$ and $\ell_{2}$ applied at each of the boundaries. The disturbance equations for linear axisymmetric stability are considered. In the succeeding section, an abstract formulation of the stability problem is made. A proof is given that for all nonnegative inner slip length $\ell_{1}$, but for sufficiently small positive outer slip length, when this slip $\ell_{2}$ is less than the radius of the outer cylinder, linear stability to axisymmetric disturbances persists. Then we have the more refined condition, no instability when

$$
\Omega_{1}>\Omega_{2}, \Omega_{2} r_{2}^{2}>\Omega_{1} r_{1}^{2} \text {, and } \ell_{2}<r_{2} \text { for all } \ell_{1} \geq 0 .
$$

Following that, in section 4. exchange of stabilities is sought and a proof is provided that stability occurs when a "slip" Taylor number is zero. Then, a numerical study is presented. Two regimes are considered in detail: (i) for no-slip on the inner cylinder with slip on the outer cylinder, and (ii) for equal slip on both. In each case it is confirmed that for sufficiently small positive slip length, when the slip $\ell_{2}$ on the outer cylinder is less than some critical value, linear stability to axisymmetric disturbances persists. When an instability does occur, the principle of exchange of stabilities (PES) emerges for these flows for all slip lengths treated. The rotation rates of the cylinders are not completely determinative of the instability. It is found that instability occurs to axisymmetric disturbances in what is normally termed the "Rayleigh-stable" regime (1.1), when the slip 
$\ell_{2}=\ell$ is greater than some critical value $\ell_{c_{1}}$. In case (i) the growth rate of the unstable mode grows with increasing $\ell$. In case (ii), with increasing $\ell$ the instability persists until it goes away beyond a second critical value of the slip length $\ell_{c_{2}}$. An explanation of these phenomena is provided in the concluding section.

\section{The governing equations.}

2.1. The equations of motion. The fluid equations are the incompressible NavierStokes equations ([8]) in cylindrical coordinates $(r, \theta, z)$, with velocity components $\left(u_{r}, u_{\theta}, u_{z}\right)$, pressure $p$, kinematic viscosity $\nu$ :

$$
\begin{gathered}
\frac{\partial u_{r}}{\partial t}+u_{r} \frac{\partial u_{r}}{\partial r}+\frac{u_{\theta}}{r} \frac{\partial u_{r}}{\partial \theta}+u_{z} \frac{\partial u_{r}}{\partial z}-\frac{u_{\theta}^{2}}{r}=-\frac{1}{\rho} \frac{\partial p}{\partial r}+\nu\left[\nabla^{2} u_{r}-\frac{u_{r}}{r^{2}}-\frac{2}{r^{2}} \frac{\partial u_{\theta}}{\partial \theta}\right] \\
\frac{\partial u_{\theta}}{\partial t}+u_{r} \frac{\partial u_{\theta}}{\partial r}+\frac{u_{\theta}}{r} \frac{\partial u_{\theta}}{\partial \theta}+u_{z} \frac{\partial u_{\theta}}{\partial z}+\frac{u_{r} u_{\theta}}{r}=-\frac{1}{\rho r} \frac{\partial p}{\partial \theta}+\nu\left[\nabla^{2} u_{\theta}-\frac{u_{\theta}}{r^{2}}+\frac{2}{r^{2}} \frac{\partial u_{r}}{\partial \theta}\right], \\
\frac{\partial u_{z}}{\partial t}+u_{r} \frac{\partial u_{z}}{\partial r}+\frac{u_{\theta}}{r} \frac{\partial u_{z}}{\partial \theta}+u_{z} \frac{\partial u_{z}}{\partial z}=-\frac{1}{\rho} \frac{\partial p}{\partial z}+\nu \nabla^{2} u_{z},
\end{gathered}
$$

where

$$
\nabla^{2} \equiv \frac{\partial^{2}}{\partial r^{2}}+\frac{1}{r} \frac{\partial}{\partial r}+\frac{1}{r^{2}} \frac{\partial^{2}}{\partial \theta^{2}}+\frac{\partial^{2}}{\partial z^{2}} .
$$

The continuity equation is:

$$
\frac{1}{r} \frac{\partial}{\partial r}\left(r u_{r}\right)+\frac{1}{r} \frac{\partial u_{\theta}}{\partial \theta}+\frac{\partial u_{z}}{\partial z}=0
$$

The Navier boundary conditions for incompressible Newtonian fluid involve the stress tensor, which is given by $\mathbb{S}=2 \mu_{v} \mathbb{D}$, where $\mu_{v}$ is the viscosity and the rate of deformation tensor is $\mathbb{D}$. Of particular interest are the components needed on the surfaces of the cylinders $([1])$ :

$$
\mathbb{D}_{r \theta}=\frac{1}{2}\left[r \frac{\partial}{\partial r}\left(\frac{u_{\theta}}{r}\right)+\frac{1}{r} \frac{\partial u_{r}}{\partial \theta}\right]
$$

and

$$
\mathbb{D}_{r z}=\frac{1}{2}\left[\frac{\partial u_{r}}{\partial z}+\frac{\partial u_{z}}{\partial r}\right] .
$$

2.2. The basic flow. Consider then, flow of a viscous incompressible fluid between coaxial rotating cylinders with radii $r_{1}$ and $r_{2}\left(r_{2}>r_{1}\right)$ with respective angular velocities $\Omega_{1}$ and $\Omega_{2}$. Assume impermeability, but also Navier slip so that the relevant boundary conditions on the basic flow are

$$
\begin{gathered}
u_{r}=u_{z}=0, \text { on } r=r_{1}, r_{2} \text { while } \\
u_{\theta}-\Omega_{1} r_{1}-\ell_{1} r \frac{\partial}{\partial r}\left(\frac{u_{\theta}-\Omega_{1} r_{1}}{r}\right)=0 \text { on } r=r_{1}, \\
u_{\theta}-\Omega_{2} r_{2}+\ell_{2} r \frac{\partial}{\partial r}\left(\frac{u_{\theta}-\Omega_{2} r_{2}}{r}\right)=0 \text { on } r=r_{2},
\end{gathered}
$$


which allows for slip on the rotating cylinders, with slip-lengths $\ell_{1}, \ell_{2}$. Notice that in calculating the stress tensor in (2.5)-(2.6), the shear is measured relative to the moving cylinders, while for $u_{z}$ the slip conditions are $u_{z} \propto \mathbb{D}_{r z}$, so that

$$
\begin{aligned}
& u_{z}-\ell_{1} \frac{\partial u_{z}}{\partial r}=0, \text { on } r=r_{1}, \\
& u_{z}+\ell_{2} \frac{\partial u_{z}}{\partial r}=0, \text { on } r=r_{2} .
\end{aligned}
$$

We look for a steady solution $(\partial / \partial t=0)$ to the equations of motion with $u_{r}=u_{z}=0$, which reduce to the ordinary differential equations

$$
\begin{aligned}
\frac{u_{\theta}^{2}}{r} & =\frac{1}{\rho} \frac{d p}{d r}, \\
\frac{d^{2} u_{\theta}}{d r^{2}}+\frac{1}{r} \frac{d u_{\theta}}{d r}-\frac{u_{\theta}}{r^{2}} & =0 .
\end{aligned}
$$

The basic circumferential velocity is therefore

$$
u_{\theta}=V(r)=A r+\frac{B}{r}, r_{1} \leq r \leq r_{2},
$$

with

$$
A=\frac{r_{2}^{2} \Omega_{2}\left(r_{2}-\ell_{2}\right)\left(r_{1}+2 \ell_{1}\right)-r_{1}^{2} \Omega_{1}\left(r_{2}-2 \ell_{2}\right)\left(r_{1}+\ell_{1}\right)}{r_{1} r_{2}\left(r_{2}^{2}-r_{1}^{2}\right)+2 \ell_{1} r_{2}^{3}+2 \ell_{2} r_{1}^{3}}
$$

and

$$
B=\frac{r_{1}^{2} r_{2}^{2}\left[r_{2} r_{1}\left(\Omega_{1}-\Omega_{2}\right)+\ell_{1} r_{2} \Omega_{1}+\ell_{2} r_{1} \Omega_{2}\right]}{r_{1} r_{2}\left(r_{2}^{2}-r_{1}^{2}\right)+2 \ell_{1} r_{2}^{3}+2 \ell_{2} r_{1}^{3}} .
$$

One notices that, as expected, when $\ell_{1}, \ell_{2} \rightarrow 0, A \rightarrow a$ and $B \rightarrow b$, the no-slip values of the coefficients. The fact that $a>0, b>0$ leads to stability when (1.1) holds in the viscous case was first demonstrated in the work of Synge ([14]), though it is called "Rayleigh's criterion". A comparison of the graphs of three Rayleigh-stable velocity distributions is given in Figure 1 with $r_{1}=5, r_{2}=15, \Omega_{1}=5, \Omega_{2}=2$. Besides no-slip, there are the slip cases $\ell_{1}=0, \ell_{2}=18.5$ and $\ell_{1}=\ell_{2}=18.5$.

2.2.1. Dimensionless parameters. The more recent approach (2, [6]) has been to scale the system so that $r_{1}=\eta$ and $r_{2}=1$, while in [14] Synge scaled the inner radius to unity. The rotation rates are taken as $\Omega_{1}$ and $\mu \Omega_{1}$, respectively. The Taylor number $\hat{T}$ is defined as

$$
\hat{T}=-\frac{4 A B}{\nu^{2}} r_{2}^{2} .
$$

The angular velocity within the fluid is $\hat{\Omega}=V(r) / r$ :

$$
\hat{\Omega}=\frac{1}{r^{2}}-\varpi, \quad \eta \leq r \leq 1,
$$

where

$$
\varpi=-\frac{A}{B} r_{2}^{2}=\frac{-\mu\left(1-\ell_{2}\right)\left(\eta+2 \ell_{1}\right)+\eta^{2}\left(1-2 \ell_{2}\right)\left(\eta+\ell_{1}\right)}{\eta^{2}\left[\eta(1-\mu)+\ell_{1}+\eta \mu \ell_{2}\right]} .
$$

We observe that $\varpi$ and $\hat{T}$ have the same sign. The scaled values of $\ell_{1}, \ell_{2}$ occur in (2.12) so that $\ell_{1}=1$ or $\ell_{2}=1$ correspond to the radius of the outer cylinder. Furthermore, if 


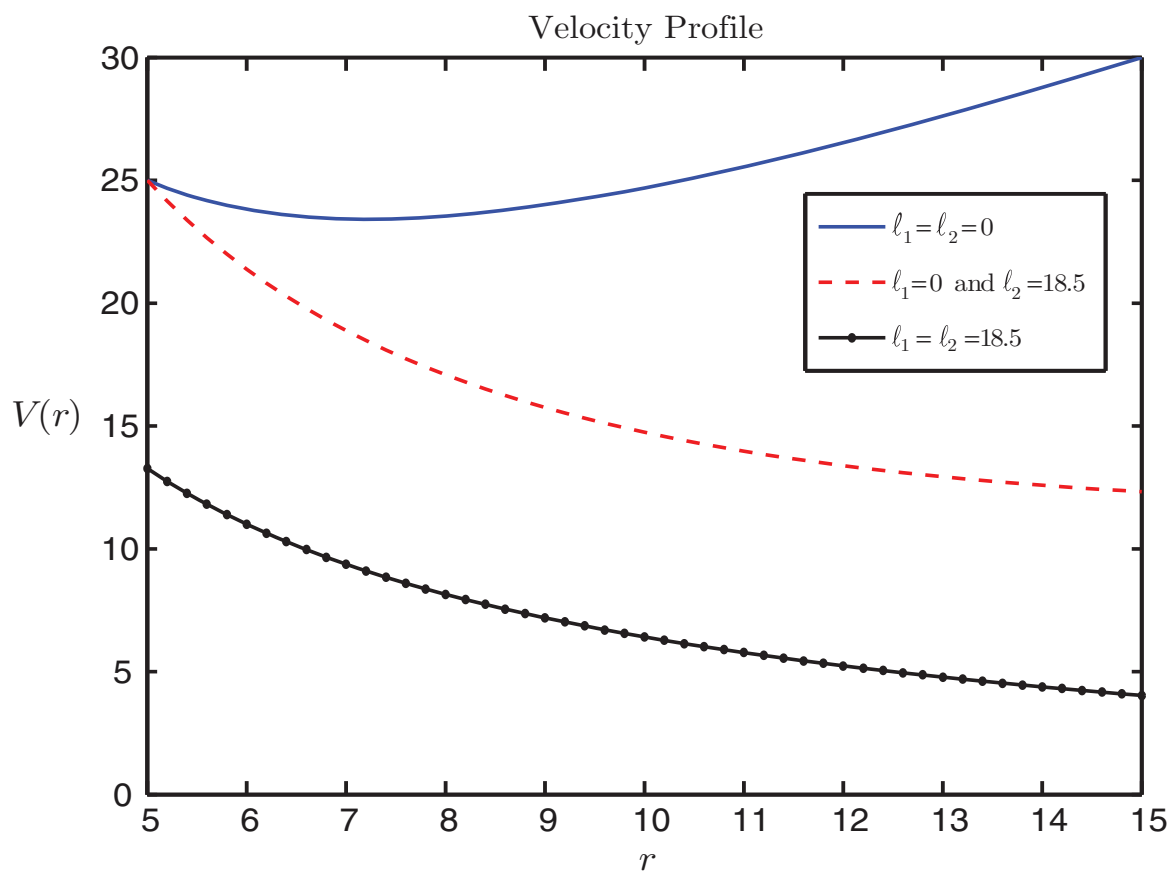

FIG. 1. $V(r)=A r+\frac{B}{r}$

$\ell_{2}=0$, then for all $\ell_{1} \geq 0$,

$$
\varpi=\frac{-\left(\eta+\ell_{1}\right)\left(\mu-\eta^{2}\right)-\mu \ell_{1}}{\eta^{2}\left[\eta(1-\mu)+\ell_{1}\right]}<0
$$

for $1>\mu>\eta^{2}$. It is for conditions such as these that the proof of stability is applicable. In the case of slip-flow we will obtain a more refined condition than (1.1): no instability when (1.2) holds.

On the other hand, if $\ell_{1}=0$, then

$$
\varpi=\frac{\left(-\mu+\eta^{2}\right)+\ell_{2}\left(\mu-2 \eta^{2}\right)}{\eta^{2}\left[1+\mu\left(\ell_{2}-1\right)\right]},
$$

and $0<\varpi \leq 1$ for an interval of values of $\ell_{2}>1$. It is for these values that an expectation of PES is relevant.

We inspect more closely the angular velocity parameter $\varpi$ as a function of $\ell_{1}$ and $\ell_{2}$. We will see in section 4.2.2 that for Case (i) with $\ell_{1}=0$, there is a first value of $\ell_{2}=\ell_{c}$ such that $\varpi\left(0, \ell_{c}\right)=0, \varpi\left(0, \ell_{2}\right)>0$ for $\ell_{2}>\ell_{c}$. Also, for Case (ii), if $\ell_{1}=\ell_{2}=\ell$, two zeros $\ell_{c_{1}}, \ell_{c_{2}}$ of $\varpi$ occur. These observations follow quite easily from the definition (2.12) but it will also be shown in section 4.2.2 that instability occurs for $\ell_{c_{1}}<\ell<\ell_{c_{2}}$. Thus the PES is pertinent for this problem. Next we derive the disturbance equations, then put them in an abstract form which readily leads to the proof of stability. In section 4 the PES is also explored. 
2.3. The disturbance equations. The linearized disturbances to be treated are also assumed to be axisymmetric and periodic in the axial direction yielding only the variables $u_{r}$ and $u_{\theta}$ after employing the equation of continuity. The velocity components are separated as

$$
\left(\begin{array}{l}
u_{r}(r, z, t) \\
u_{\theta}(r, z, t)
\end{array}\right)=\left(\begin{array}{c}
u(r) \\
v(r)
\end{array}\right) e^{i k z+\sigma t},
$$

and the nondimensional wave number is $k$ and the growth rate of disturbances is $\sigma$. Then the pair of equations become, in the current notation,

$$
\begin{gathered}
\left(D D_{*}-k^{2}-\sigma\right)\left(D D_{*}-k^{2}\right) u=-k^{2} \hat{T} \hat{\Omega} v \\
\left(D D_{*}-k^{2}-\sigma\right) v=u
\end{gathered}
$$

where $D=d / d r, D_{*}=D+1 / r$.

A unique feature of this work is the set of boundary conditions which apply. We have already seen (2.5)-(2.6), which lead to

$$
\begin{aligned}
& v-\ell_{1} r \frac{\partial}{\partial r}\left(\frac{v}{r}\right)=0 \text { on } r=\eta, \\
& v+\ell_{2} r \frac{\partial}{\partial r}\left(\frac{v}{r}\right)=0 \text { on } r=1,
\end{aligned}
$$

for the azimuthal velocity perturbation. With the no normal flow condition we obtain

$$
u=0, \text { on } r=\eta, r=1,
$$

but slip also appears from the stress conditions so that

$$
\begin{aligned}
& \ell_{1} D D_{*} u-D_{*} u=0 \text { on } r=\eta, \\
& \ell_{2} D D_{*} u+D_{*} u=0 \text { on } r=1 .
\end{aligned}
$$

Combining (2.20) with (2.21), (2.22) we find that they simplify to

$$
\begin{aligned}
& \ell_{1} D_{*} D u-D u=0 \text { on } r=\eta, \\
& \ell_{2} D_{*} D u+D u=0 \text { on } r=1 .
\end{aligned}
$$

\section{Abstract formulation.}

3.1. The underlying operators. One important consideration is a derivation of conditions under which stability holds. We will first look at parametric conditions that will ensure this. To show how (1.2) comes about, an operator notation is introduced, which clarifies the nature of the analysis. The system (2.16)-(2.17) thereby becomes

$$
\begin{aligned}
(\hat{M}+\sigma) M_{0} u & =-k^{2} \hat{T} \hat{\Omega} v, \\
\left(M_{\ell}+\sigma\right) v & =-u .
\end{aligned}
$$

In this notation, $M_{0}, \hat{M}$ and $M_{\ell}$ all operate in the same way as the differential operator $M^{*}:=-D D_{*}+k^{2}$, but are considered different operators because of the distinct boundary conditions satisfied by the functions on which they act, while $\hat{M} M_{0}$ operates as $\left(M^{*}\right)^{2}=\left(-D D_{*}+k^{2}\right)^{2}$. It is also understood that the functions have the necessary differentiability properties ([6]). Thus, $M^{*}$ assumes no boundary conditions, $M_{0}$ acts on functions that have the same boundary conditions as $u$ [eq. (2.20)], $\hat{M} M_{0}$ assumes that 
the functions satisfy boundary conditions (2.20) and (2.23)-(2.24), whereas $M_{\ell}$ uses the boundary conditions of $v$ [eqs. (2.18), (2.19)]. Notice that $M_{0}$ corresponds to $\ell_{1}=\ell_{2}=0$.

Introduce an inner product,

$$
\langle f, g\rangle=\int_{\eta}^{1} r f(r) \bar{g}(r) d r, f, g \in \mathfrak{H},
$$

in which the overbar denotes complex conjugation, where

$$
\mathfrak{H}=\left\{\left.\varphi\left|\int_{\eta}^{1} r\right| \varphi\right|^{2} d r<\infty\right\}
$$

and norm

$$
\|\varphi\|=\langle\varphi, \varphi\rangle^{1 / 2} .
$$

The differential operators $M_{0}, \hat{M} M_{0}$, and $M_{\ell}$ all have the property of being selfadjoint in this inner product ([10]). This indicates that in fact, $\hat{M} M_{0}=M_{0} \hat{M}^{*}$, explaining why the operator is selfadjoint. The fact that $M_{0}$ is positive definite is straightforward. A lengthier calculation can show the same for $\hat{M} M_{0}$. However, it will be shown that $\hat{M} M_{0}=M_{0} P M_{0}$, for a certain bounded positive-definite operator $P$. One objective then is to determine the definiteness condition for $M_{\ell}$, so that in fact $M_{0}$ agrees with $M_{\ell}$, when $\ell_{1}=\ell_{2}=0$. The details of the factoring of these operators now follow.

3.2. The operator factors. As suggested by equation (3.1), we make the change of variables

$$
\zeta=M_{0} \varphi
$$

which is explicitly

$$
\zeta=\left(-\frac{d}{d r}\left(\frac{d}{d r}+\frac{1}{r}\right)+k^{2}\right) \varphi, \varphi \in \operatorname{dom} M_{0} .
$$

The boundary conditions on $\varphi$ are (2.20). However, there are no simple boundary conditions on $\zeta$ on the walls. We make use of the representation

$$
\zeta=M_{0} \varphi \Rightarrow \varphi=M_{0}^{-1} \zeta
$$

The operator $M_{0}^{-1}$ is an integral operator with a Green's function $g(r, s)$ so that

$$
\varphi(r)=\int_{\eta}^{1} g(r, s) \zeta(s) d s .
$$

Now employing the boundary conditions (2.23)-(2.24) on (3.5), we have

$$
\begin{aligned}
& \ell_{2} \zeta(1)-\int_{\eta}^{1} g_{r}(1, s) \zeta(s) d s=0, \quad \ell_{2}>0, \\
& \ell_{1} \zeta(\eta)+\int_{\eta}^{1} g_{r}(\eta, s) \zeta(s) d s=0, \quad \ell_{1}>0 .
\end{aligned}
$$

Next we provide an explicit calculation ([7]) of the Green's function $g(r, s)$. It satisfies

$$
M_{0} g=\delta(r-s)
$$

or

$$
-\frac{d}{d r}\left(\frac{1}{r} \frac{d}{d r}(r g)\right)+k^{2} g=\delta(r-s) .
$$


Since a fundamental pair of solutions to $M^{*} g=0$ is $I_{1}(k r)$ and $K_{1}(k r)$, involving the modified Bessel functions of order 1, then

$$
\begin{aligned}
g(r, s) & =\frac{\left[K_{1}(k \eta) I_{1}(k s)-I_{1}(k \eta) K_{1}(k s)\right]\left[I_{1}(k) K_{1}(k r)-K_{1}(k) I_{1}(k r)\right]}{K_{1}(k \eta) I_{1}(k)-I_{1}(k \eta) K_{1}(k)}, \quad r>s, \\
& =g(s, r), \quad r<s .
\end{aligned}
$$

It also follows that because $K_{1}(k r)$ is decreasing and $I_{1}(k r)$ is increasing on $\eta \leq r, s \leq$ 1 , the Green's function is nonnegative: $g(r, s) \geq 0$. Furthermore, the terms needed in the integrands in (3.6)-(3.7) turn out to be simply

$$
g_{r}(1, s)=-\frac{K_{1}(k \eta) I_{1}(k s)-I_{1}(k \eta) K_{1}(k s)}{K_{1}(k \eta) I_{1}(k)-I_{1}(k \eta) K_{1}(k)}
$$

and

$$
g_{r}(\eta, s)=\frac{K_{1}(k s) I_{1}(k)-I_{1}(k s) K_{1}(k)}{\eta\left[K_{1}(k \eta) I_{1}(k)-I_{1}(k \eta) K_{1}(k)\right]} .
$$

The operator $\hat{M}$ in (3.1) may thus be defined as

$$
\hat{M} \zeta=\left(-\frac{d}{d r}\left(\frac{d}{d r}+\frac{1}{r}\right)+k^{2}\right) \zeta, \eta<r<1,
$$

and conditions satisfied by $\zeta$ are (3.6)-(3.7). This formulation also permits the specification of the operator $P$ alluded to earlier. It is found by applying $M_{0}^{-1}$ to $\hat{M} \zeta$ so that

$$
\begin{aligned}
P \zeta & :=M_{0}^{-1} \hat{M} \zeta=\int_{\eta}^{1} g(r, s)\left(-\frac{d}{d s}\left(\frac{d}{d s}+\frac{1}{s}\right)+k^{2}\right) \zeta d s \\
& =\left[g_{s}(r, s) \zeta(s)\right]_{s=\eta}^{1}+\int_{\eta}^{1} \zeta(s) \delta(r-s) d s .
\end{aligned}
$$

After applying (3.6), (3.7) and evaluating the terms involving $g_{s}(r, s)$, the result is

$$
P \zeta=\zeta(r)+\int_{\eta}^{1}\left[\frac{1}{\ell_{2}} g_{r}(1, s) g_{s}(r, 1)+\frac{1}{\ell_{1}} g_{r}(\eta, s) g_{s}(r, \eta)\right] \zeta(s) d s, \ell_{1}, \ell_{2}>0,
$$

when $\zeta \in \operatorname{dom} \hat{M}$. We note that

$$
g_{s}(r, 1)=-\frac{K_{1}(k \eta) I_{1}(k r)-I_{1}(k \eta) K_{1}(k r)}{K_{1}(k \eta) I_{1}(k)-I_{1}(k \eta) K_{1}(k)}
$$

and

$$
g_{s}(r, \eta)=\frac{I_{1}(k) K_{1}(k r)-K_{1}(k) I_{1}(k r)}{\eta\left[K_{1}(k \eta) I_{1}(k)-I_{1}(k \eta) K_{1}(k)\right]} .
$$

By this means, we observe that $P$ is bounded, symmetric and positive definite. It may therefore be applied to any $f \in \mathfrak{H}$. We may therefore express the system (3.1)-(3.2) as

$$
\begin{aligned}
\left(M_{0} P M_{0}+\sigma M_{0}\right) u & =-k^{2} \hat{T} \hat{\Omega} v, \\
\left(M_{\ell}+\sigma\right) v & =-u .
\end{aligned}
$$


3.3. The equations in symmetrized form. If we write (3.10), 3.11) in matrix form we see that

$$
\left(\begin{array}{cc}
M_{0} P M_{0} & \hat{T} \hat{\Omega} k^{2} \\
1 & M_{\ell}
\end{array}\right)\left(\begin{array}{l}
u \\
v
\end{array}\right)=-\sigma\left(\begin{array}{cc}
M_{0} & 0 \\
0 & 1
\end{array}\right)\left(\begin{array}{l}
u \\
v
\end{array}\right) .
$$

It is possible to write (3.12) with more symmetry as

$$
\begin{aligned}
& \left(\begin{array}{cc}
M_{0} P M_{0} & \hat{T} \hat{\Omega} k^{2} \\
-\hat{T} \hat{\Omega} k^{2} & -\hat{T} \hat{\Omega} k^{2} M_{\ell}
\end{array}\right)\left(\begin{array}{l}
u \\
v
\end{array}\right) \\
& =-\sigma\left(\begin{array}{cc}
M_{0} & 0 \\
0 & -\hat{T} \hat{\Omega} k^{2}
\end{array}\right)\left(\begin{array}{l}
u \\
v
\end{array}\right) .
\end{aligned}
$$

We are therefore led to consider

$$
(\mathcal{L} \Phi, \Phi)=-\sigma(\mathfrak{M} \Phi, \Phi)
$$

where

$$
\begin{aligned}
\Phi & =\left(\begin{array}{l}
u \\
v
\end{array}\right), \\
\mathcal{L} & =\left(\begin{array}{cc}
M_{0} P M_{0} & -W(r) \\
W(r) & W(r) M_{\ell}
\end{array}\right), \\
\mathfrak{M} & =\left(\begin{array}{cc}
M_{0} & 0 \\
0 & W(r)
\end{array}\right), W(r)=-\hat{T} \hat{\Omega} k^{2} .
\end{aligned}
$$

In general, if one examines the approach taken by Chandrasekhar ([2]) and proceeds almost identically, nonzero boundary terms appear. These terms may be handled without much difficulty.

The main difficulty this causes is establishing that

$$
\operatorname{Re}\left\langle M_{\ell} v, W(r) v\right\rangle=\operatorname{Re}\left\{\int_{\eta}^{1}\left(-D D_{*}+k^{2}\right) v(W \bar{v} r) d r\right\}>0
$$

which was proved to be true if the boundary conditions are no-slip $\left(\left[7\right.\right.$, p.80]), i.e. $\ell_{1}=$ $\ell_{2}=0$.

Incidentally, if $W$ is set to unity, the boundary conditions (2.18)-(2.19) lead to

$$
\begin{aligned}
& \left\langle M_{\ell} v, v\right\rangle=\int_{\eta}^{1}\left(-D D_{*}+k^{2}\right) v \bar{v} r d r=\int_{\eta}^{1}\left[-\frac{1}{r^{2}} D\left(r^{3} D\left(\frac{v}{r}\right)\right) \bar{v} r+k^{2} r|v|^{2}\right] d r \\
& =\int_{\eta}^{1}\left(r^{3}\left|\left(\frac{v}{r}\right)^{\prime}\right|^{2}+k^{2} r|v|^{2}\right) d r+\ell_{2}\left|\left(\frac{v}{r}\right)^{\prime}(1)\right|^{2}+\ell_{1} \eta^{3}\left|\left(\frac{v}{r}\right)^{\prime}(\eta)\right|^{2}>0
\end{aligned}
$$

Thus, we have proved the following:

LEmma 1. The selfadjoint operator $M_{\ell}$ is positive definite for all $\ell_{1}, \ell_{2} \geq 0$.

Theorem 1. The flow (2.11) is stable, that is, $\operatorname{Re}(\sigma)<0$, when $0 \leq \ell_{2} \leq 1$ and $\hat{T}<0(\varpi<0)$, for all $\ell_{1} \geq 0$. 
3.4. Proof of stability.

Proof. Using Chandrasekhar's approach consider

$$
\begin{aligned}
\operatorname{Re} & \int_{\eta}^{1}\left(-D D_{*}+k^{2}\right) v W \bar{v} r d r \\
= & \operatorname{Re} \int_{\eta}^{1}\left[-\frac{d}{d r}\left(r \frac{d}{d r}\right)+\frac{1}{r}+k^{2} r\right] v W \bar{v} d r \\
= & \operatorname{Re}\left\{-\left.r v^{\prime} W \bar{v}\right|_{\eta} ^{1}+\int_{\eta}^{1} r v^{\prime} W^{\prime} \bar{v} d r\right\} \\
& +\int_{\eta}^{1} W(r)\left(r\left|v^{\prime}\right|^{2}+\frac{|v|^{2}}{r}+k^{2} r|v|^{2}\right) d r .
\end{aligned}
$$

Since

$$
\begin{gathered}
W(r)=-\hat{T} k^{2}\left(\frac{1}{r^{2}}-\varpi\right), \\
W^{\prime}(r)=\frac{2 \hat{T} k^{2}}{r^{3}} .
\end{gathered}
$$

Thus

$$
\operatorname{Re} \int_{\eta}^{1} r v^{\prime} W^{\prime} \bar{v} d r=\frac{1}{2} \int_{\eta}^{1} r W^{\prime} \frac{d}{d r}|v|^{2} d r=\int_{\eta}^{1}\left(\hat{T} k^{2} \varpi-W\right) \frac{d}{d r}|v|^{2} d r .
$$

The form (3.20) becomes

$$
\begin{aligned}
\operatorname{Re} & \int_{\eta}^{1}\left(-D D_{*}+k^{2}\right) v W \bar{v} r d r \\
= & \int_{\eta}^{1} r W\left(\left|v^{\prime}-\frac{v}{r}\right|^{2}+k^{2}|v|^{2}\right) d r \\
& -\left[\frac{r W}{2}\left(v^{\prime} \bar{v}+v \bar{v}^{\prime}\right)\right]_{\eta}^{1}+\left.\hat{T} k^{2} \varpi|v|^{2}\right|_{\eta} ^{1} .
\end{aligned}
$$

We abbreviate the boundary conditions (2.18) and (2.19) as

$$
\begin{aligned}
& v(\eta)=\gamma_{1} v^{\prime}(\eta), \\
& v(1)=-\gamma_{2} v^{\prime}(1),
\end{aligned}
$$

where $\gamma_{1}, \gamma_{2}$ are the appropriate constants. That is,

$$
\gamma_{1}=\frac{\ell_{1}}{1+\frac{\ell_{1}}{\eta}}
$$

and

$$
\gamma_{2}=\frac{\ell_{2}}{1-\ell_{2}}
$$

When applied to the boundary terms in (3.21), the result is

$$
W(1)\left(\frac{1}{\gamma_{2}}\right)|v(1)|^{2}+W(\eta)\left(\frac{\eta}{\gamma_{1}}\right)|v(\eta)|^{2}+\hat{T} k^{2} \varpi|v(1)|^{2}-\hat{T} k^{2} \varpi|v(\eta)|^{2} .
$$


So whenever $\hat{T}<0, W(r)>0, \hat{T} k^{2} \varpi>0$, and a calculation shows that

$$
W(\eta)\left(\frac{\eta}{\gamma_{1}}\right)>\hat{T} k^{2} \varpi .
$$

When $0 \leq \ell_{2} \leq 1$, then $\gamma_{2}>0$ so that (3.24) is positive. In particular when $\ell_{2}=0$, (3.25) is satisfied by (2.13).

Complete the analysis of (3.14)-(3.17). Noting that $\mathfrak{M}$ is positive definite in the inner product

$$
(\Phi, \Psi)=\left\langle\Phi_{1}, \Psi_{1}\right\rangle+\left\langle\Phi_{2}, \Psi_{2}\right\rangle
$$

because $W(r)>0$,

$$
(\mathfrak{M} \Phi, \Phi)=\left\langle M_{0} u, u\right\rangle+\langle W v, v\rangle>0,
$$

we are also able to confirm under what conditions $\operatorname{Re}(\mathcal{L} \Phi, \Phi)>0$, that is, $\mathcal{L}$ is accretive [10, p.279]. Explicitly, we see that

$$
\begin{aligned}
& (\mathcal{L} \Phi, \Phi)=\left\langle M_{0} P M_{0} u, u\right\rangle-\langle W v, u\rangle+\langle W u, v\rangle+\left\langle W M_{\ell} v, v\right\rangle, \\
& \text { complex conjugates }
\end{aligned}
$$

and thus, taking real parts we have

$$
\operatorname{Re}(\mathcal{L} \Phi, \Phi)=\left\langle P M_{0} u, M_{0} u\right\rangle+\operatorname{Re}\left\langle W M_{\ell} v, v\right\rangle=-\operatorname{Re}(\sigma)(\mathfrak{M} \Phi, \Phi) .
$$

We have shown above in (3.21) that under suitable conditions (1.2), $\operatorname{Re}\left\langle M_{\ell} v, W v\right\rangle>$ 0 . Hence $\operatorname{Re}(\sigma)<0$; the stability then follows.

Corollary 1. The flow (2.11) is always stable with no-slip on the outer cylinder.

\section{Exchange of stabilities.}

4.1. Basic statement. The regimes we have been studying up to now have been shown to be stable. However, we will see that instability will occur for $\ell_{2}$ sufficiently large. When instability does occur it is very useful to know when the most unstable eigenvalue may be found to bifurcate as a real eigenvalue from the state where $\sigma=0$. This is the principle of exchange of stabilities (PES) which in the early years made it much easier to uncover the instability. As the theory has developed, this is also vital in describing the approach to nonlinear instability as well in the "Rayleigh-unstable" case where $\mu<\eta^{2}$ 6]. However, because this work involves an instability primarily associated with slip, we are interested in the case where $\mu>\eta^{2}$, and $A<0$ in (2.8), while $B>0$ in (2.9); still the Taylor number $\hat{T}>0$. Likewise, the angular velocity parameter $\varpi>0$, for example as in (2.14). To understand the importance of this we make the following observations based on Lemma 1 .

REMARK $1 . M_{0}$ is positive definite, that is,

$$
\left\langle M_{0} \varphi, \varphi\right\rangle \geq k^{2}\|\varphi\|^{2}, \quad \varphi \in \operatorname{dom} M_{0}, \quad k \neq 0 .
$$

The case $k=0$ is excluded because stability is known to hold in this case.

REMARK 2. The resolvent operator $\Gamma_{\ell}(\sigma)=\left(M_{\ell}+\sigma\right)^{-1}, \ell_{1}, \ell_{2} \geq 0$ exists for

$$
\sigma \notin \Sigma_{k}=\left\{\sigma \in \mathbf{C} \mid \operatorname{Re}(\sigma) \leq-k^{2}, \operatorname{Im}(\sigma)=0\right\},
$$

and $\left\|\Gamma_{\ell}(\sigma)\right\|^{-1}>\left|k^{2}\right|$, for $\operatorname{Re}(\sigma)>-k^{2}$ [10, p.272]. 
We are able to make the following determination about the stability behavior at $\hat{T}=0$.

Theorem 2. The eigenvalue spectrum of (3.1) $-(3.2)$ is real and stable when $\hat{T}=0(\varpi=$ $0)$, for all $\ell_{1}, \ell_{2} \geq 0$.

Proof. Suppose $\hat{T}=0$. Then by (3.10)-(3.11), the original system (3.1)-(3.2) reduces to

$$
\begin{aligned}
\left(M_{0} P M_{0}+\sigma M_{0}\right) u & =0, \\
u+\left(M_{\ell}+\sigma\right) v & =0 .
\end{aligned}
$$

Taking the inner products of (4.1) with $u$ we see that

$$
\left\langle M_{0} P M_{0} u, u\right\rangle=-\sigma\left\langle M_{0} u, u\right\rangle .
$$

The operator $P$ is bounded symmetric and positive definite (with bound $p$ ), so

$$
\begin{aligned}
-\sigma\left\langle M_{0} u, u\right\rangle & =\left\langle M_{0} P M_{0} u, u\right\rangle=\left\langle P M_{0} u, M_{0} u\right\rangle \geq p\left\|M_{0} u\right\|^{2} \\
& \geq p\left\langle M_{0} u, u\right\rangle^{2} /\|u\|^{2} \geq p k^{2}\left\langle M_{0} u, u\right\rangle>0 .
\end{aligned}
$$

Thus $\sigma$ is real and $\sigma \leq-p k^{2}$. Hence when $\sigma \notin \Sigma_{k}, \Longrightarrow u=0 \Longrightarrow v=0$, by (4.2) and Remark 2,

We have thus shown that the disturbance equations suggest that the principle of exchange of stabilities should be valid. The numerical results to follow confirm that for positive slip-lengths on each cylinder, "the most unstable mode arises with a real eigenvalue crossing through $0 "$ ".

4.2. Numerical analysis of the instability.

4.2.1. Discretization of the disturbance equations. We now present the study of the growth rate $\sigma$ in the disturbance equations (2.16)-(2.17).

In order to do this we discretized the equations using the popular polynomial Chebyshev pseudo-spectral methods (see for instance [19] and [15). After the discretization we obtained a generalized eigenvalue problem of the form

$$
\hat{\sigma} \mathcal{B} \chi=\mathcal{A} \chi,
$$

where $\hat{\sigma}$ is an eigenvalue and $\chi$ is an eigenvector. For sufficiently fine discretization we have $\hat{\sigma} \approx \sigma$. For an instability we required that at least one eigenvalue, $\hat{\sigma}$, have positive real part.

4.2.2. Instability under the classical Rayleigh stability criterion. Two flow regimes were considered: first, slip on the outer cylinder with no-slip on the inner cylinder; second, with equal slip on both cylinders. In both cases we set $\mu=\frac{2}{5}, \eta=\frac{1}{3}$ and let the wavenumber $k=\pi$. These parameters produce a stable flow in the case of no-slip. As we shall see this is not what happens when the Navier conditions are introduced.

Case (i): Slip on outer cylinder, no-slip on inner

For this first case we set the slip of the cylinder to $\ell_{1}=0$ and vary $\ell_{2}$. In Figure 2 we can see that as $\ell_{2}$ increases and we fix all other quantities, an unstable mode arises after $\hat{T}$ passes through $\hat{T}_{\text {crit }} \approx 2819$. This first unstable mode has imaginary part equal to zero, which exemplifies the principle exchange of stabilities (PES). As $\ell_{2} \rightarrow \infty$ we have that the instability persists with increasing $\hat{T}$. 
Exchange of Stabilities

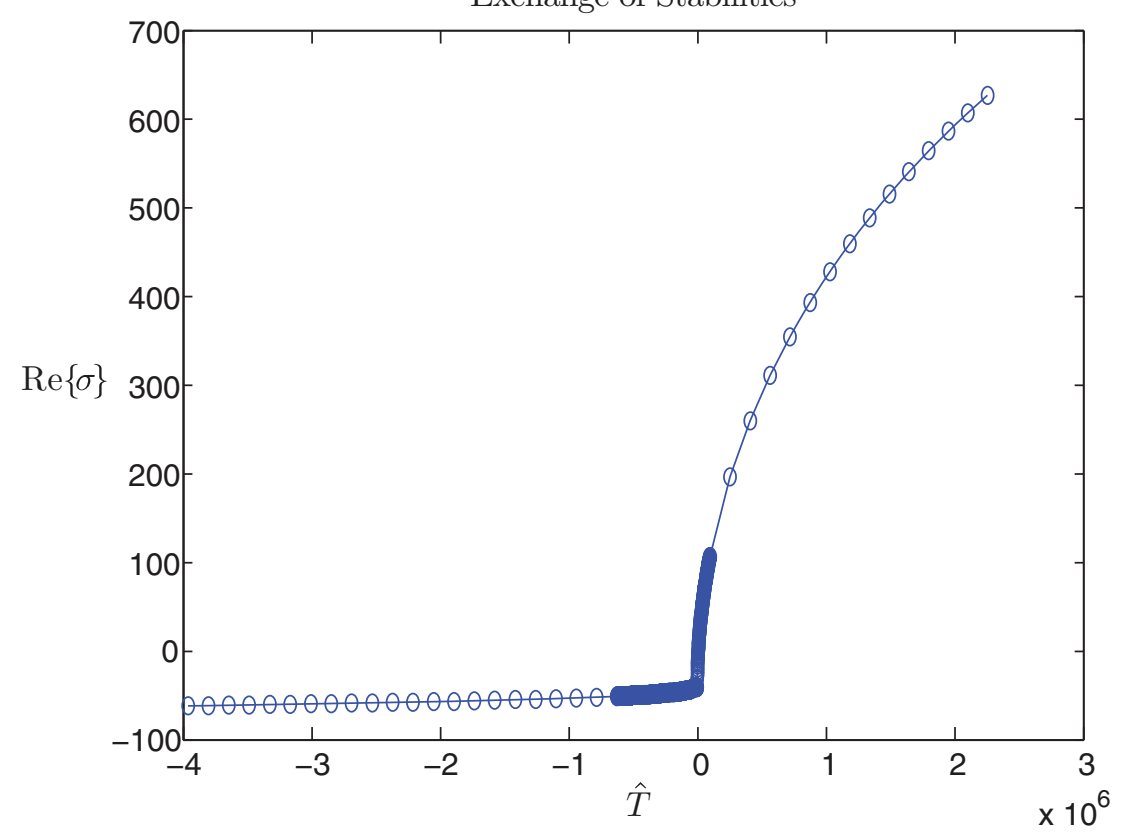

$\hat{T}$ increases with $\ell_{2}$

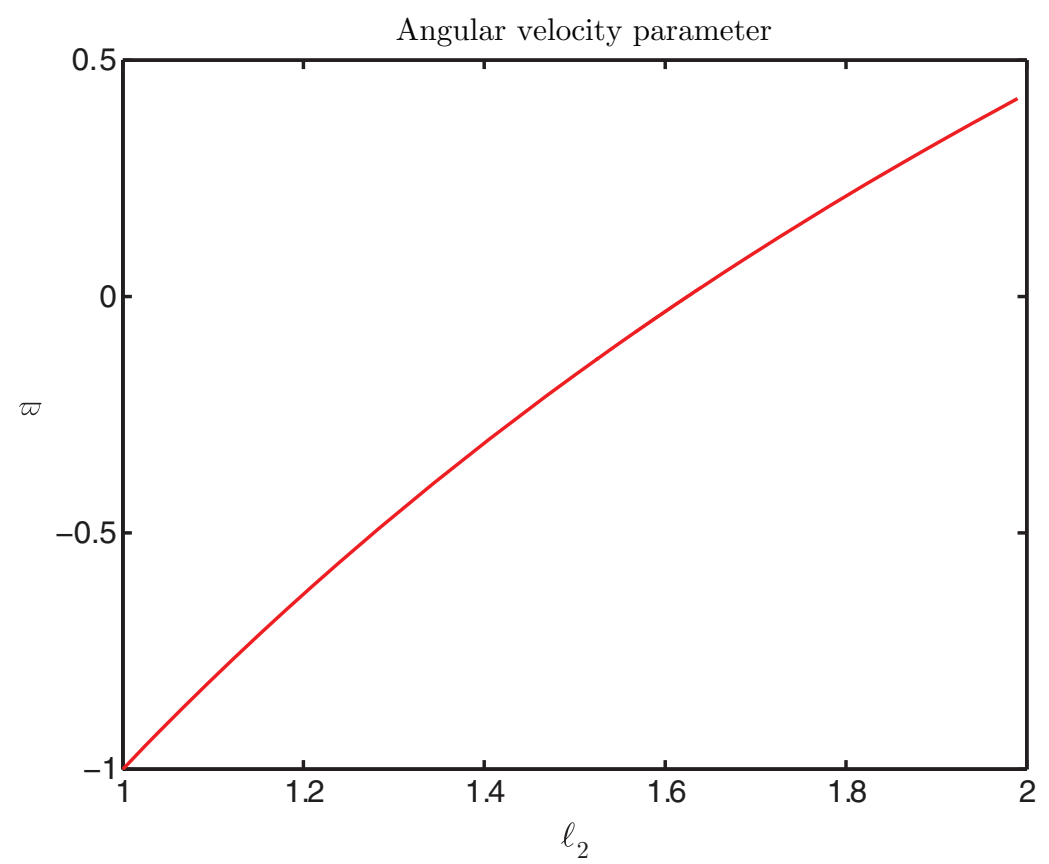

$\varpi$ bounded as $\ell_{2}$ increases

FIG. 2. Case (i): Slip on outer cylinder, no-slip on inner 


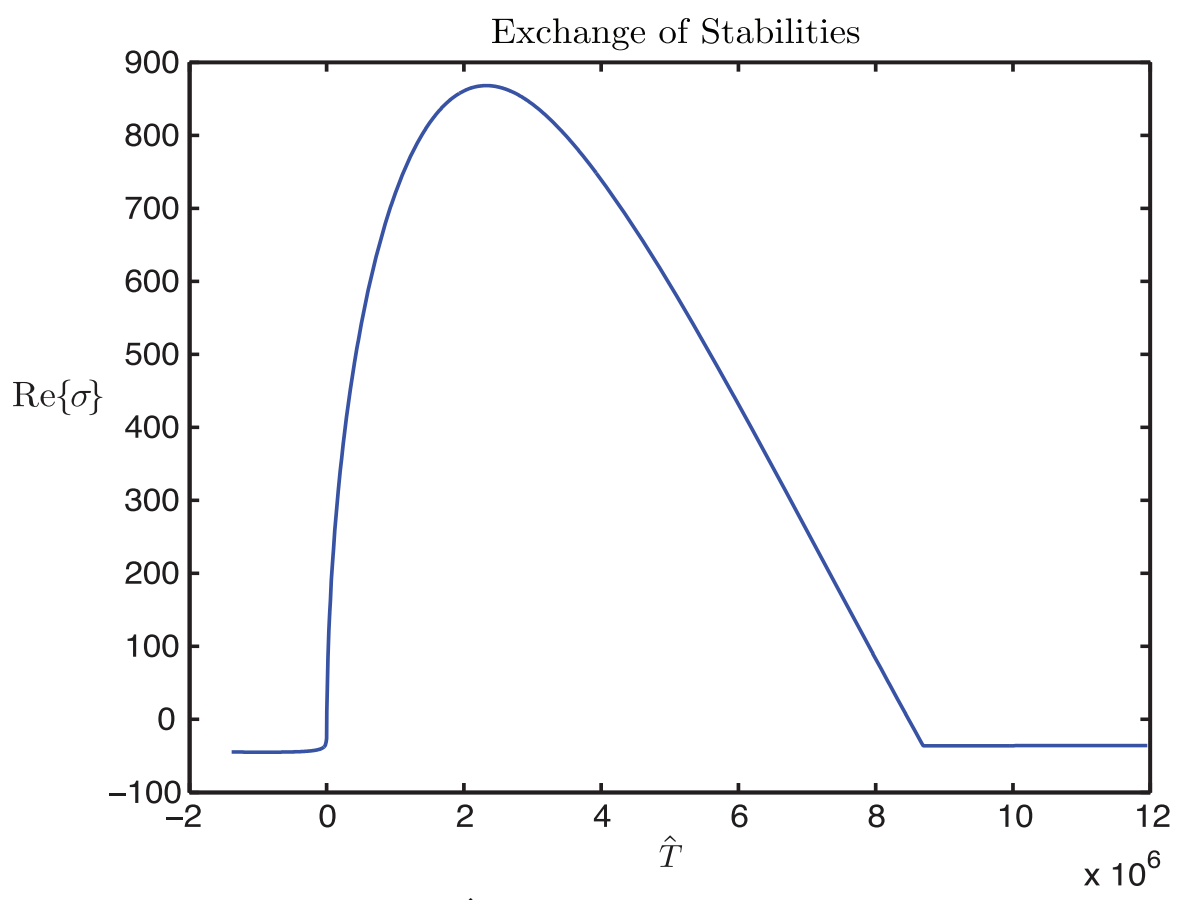

$\hat{T}$ increases with $\ell$

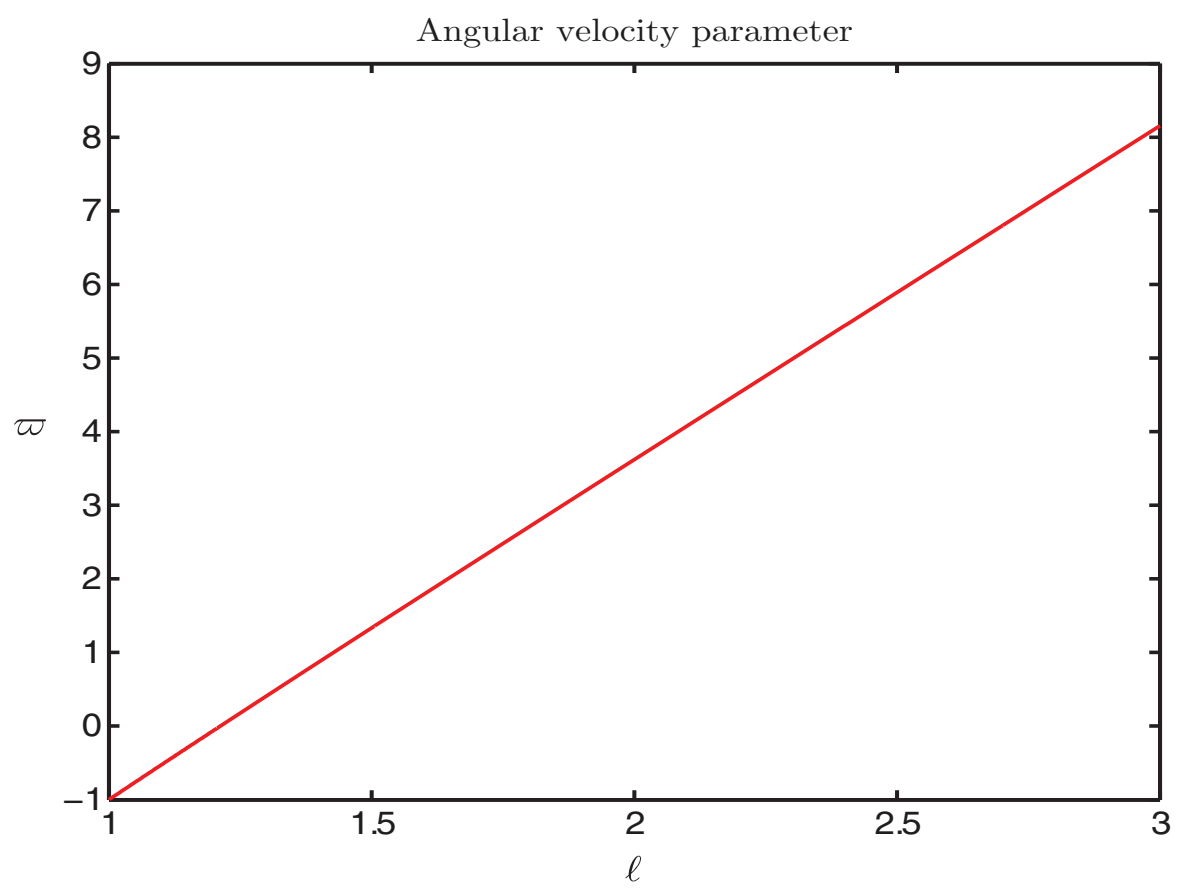

$\varpi$ grows linearly with $\ell$

FIG. 3. Case (ii): Equal slip on both cylinders 
Case (ii): Equal slip on both cylinders

We next consider the case where $\ell_{1}=\ell_{2}$. In Figure 3 again we see that instability ensues for sufficiently large slip length, also with PES. Explicitly $\hat{T}_{\text {crit }} \approx 816.2$. As opposed to the other case, having $\ell_{1} \neq 0$ helps to stabilize the flow. As $\ell_{2}$ increases, the growth rate goes negative and the flow is rendered stable. An explanation of these phenomena is provided in the next section.

5. Concluding comments. This article has presented an analysis of the flow between long rotating cylinders under the assumption of Navier slip conditions. Two regimes were solved in detail. First, there was no-slip on the inner cylinder, with slip on the outer. Second, the slip length was taken to be the same for both. Consequently, there is one slip length $\ell$ in each case. In Case (i) $\ell_{2}=\ell$, while in Case (ii) $\ell_{2}=\ell_{1}=\ell$. We found the very interesting conclusions shown in Figures 2 and 3 . In Case (i) the growth rate of the unstable mode increases indefinitely with slip length, while in Case (ii), there is a band of instability for slip lengths $\ell_{c_{1}}<\ell<\ell_{c_{2}}$.

The occurrence of these phenomena can be explained on the basis of Rayleigh's criterion ([9, p.139], after [3]), using the angular velocity distribution (2.11)

$$
\hat{\Omega}=\frac{\hat{V}(r)}{r}=\frac{1}{r^{2}}-\varpi,
$$

the angular momentum $\Lambda=r^{2} \hat{\Omega}$, and local vorticity

$$
\zeta=\frac{1}{r} \frac{d}{d r}(\Lambda)
$$

The classical criterion asserts that axisymmetric rotating flows are inviscidly unstable when the angular momentum $\Lambda$ is a decreasing function of distance from the axis of rotation so that

$$
\partial\left(\Lambda^{2}\right) / \partial r=2 r^{3} \hat{\Omega} \zeta<0,
$$

which occurs when $\hat{\Omega} \zeta<0$. Conversely, the rotating flow is inviscidly stable if $\hat{\Omega}$ and $\zeta$ have the same sign. So for the Couette flows considered here,

$$
\hat{\Omega} \zeta=-2 \varpi\left(\frac{1}{r^{2}}-\varpi\right) .
$$

We observe then that instability is expected with increasing $\ell$ : when $\hat{T}(\ell)$ increases from zero, $\varpi(\ell)$ increases from zero, which occurs when $\ell$ goes through $\ell_{c_{1}}$. Our calculations in section 4.2 .2 revealed that $\ell_{c_{1}}$ gives the values at which $\hat{T}=\hat{T}_{\text {crit }}$ and $\varpi=\varpi_{\text {crit }}$ for instability. In Case (i) we see from (2.12) that for the set of parameter values used in the calculations, $\varpi<1$, which is similar to the no-slip case. We found $\hat{T}_{\text {crit }} \approx 2819$. However in Case (ii), where we found $\hat{T}_{\text {crit }} \approx 816.2$, as $\ell$ increases, $\varpi$ increases from $\varpi_{\text {crit }}$ until $\varpi$ exceeds a second critical value, that is, when $\ell=\ell_{c_{2}}$ and instability disappears! This is a viscous flow calculation, and in [9, p. 139] these restorative effects are further discussed. Eventually, with increasing $\ell, \hat{\Omega} \zeta>0$.

By the corollary to Theorem 10 without slip on the outer cylinder no such instability occurs, which applies in this case for all positive slip lengths on the inner cylinder. Work by other authors [17, [18] has addressed this and a related problem. First see [17, 
when the inner cylinder is fixed and the outer has no slip. No instability was uncovered because the slip velocity is only on the inner cylinder. Secondly see [18, where the outer cylinder was fixed with no slip but with slip on the inner cylinder. The mode of instability uncovered in our work was not discovered in [18, because that was the Rayleigh unstable regime. Thus, though the work presented here was not experimentally driven, it might be worth investigating by the type of apparatus used in the studies of [17. and [18. There, the slip boundary condition was verified, but not as the primary agent of the instability as was discovered here.

There are other possible directions in which this research will likely proceed. One is to consider counter-rotating cases where $\mu<0$ and fully asymmetric disturbances are to be expected and contribute to a possible Hopf bifurcation. Thus the flow in both co-rotating and counter-rotating cylinders should be analyzed in the nonlinear regime.

\section{REFERENCES}

[1] Batchelor, G.K. An Introduction to Fluid Dynamics. Cambridge University Press, 1967. MR1744638 (2000j:76001)

[2] Chandrasekhar, S. Hydrodynamic and Hydromagnetic Stability. Oxford University Press, 1961. MR0128226(23:B1270)

[3] Coles, D. Transition in circular Couette flow J. Fluid Mech., 1965, 21, 385-425.

[4] Conlisk, A. T. Essentials of Micro and Nanofluidics with Applications to the Biological and Chemical Sciences. Cambridge University Press, 2011.

[5] Drazin, P. G. and Reid, W. H. Hydrodynamic Stability, Second Edition. Cambridge University Press, 2004. MR2098531 (2005g:76049)

[6] Herron, I. H. and Ali, H. N. The principle of exchange of stabilities for Couette flow. Quart. Appl. Math, 2003, 61, 279-293. MR1976370 (2004c:76061)

[7] Herron, I. H. and Foster, M. R. Partial Differential Equations in Fluid Dynamics. Cambridge University Press, 2008. MR2441490 (2009i:35001)

[8] Hunt, J. N. Incompressible Fluid Dynamics. Longmans, 1964. MR0207282 (34:7098)

[9] Joseph, D.D. Stability of Fluid Motions, Vol. I. Springer Tracts in Natural Philosophy, vol. 27, Springer-Verlag, Berlin-New York, 1976. MR0449147 (56:7452)

[10] Kato, T., Perturbation Theory for Linear Operators, Second edition, Springer-Verlag, Berlin, 1976. MR0407617 (53:11389)

[11] Matthews, M. T. and Hill, J. M., Flow around nanospheres and nanocylinders, Q. J. Mech. Appl. Math., 2006, 59, 191-210. MR2219884(2006k:76041)

[12] Matthews, M. T. and Hill, J. M. Nanofluidics and the Navier boundary condition, 2008, Int. J. Nanotechnol. 5, 218-239.

[13] Orszag, S. A. Accurate solution of the Orr-Sommerfeld stability equation, J. Fluid Mech., 1971, 50, 689-703.

[14] Synge, J. L., On the stability of a viscous liquid between rotating coaxial cylinders, Proc. Roy. Soc. A, 1938, 167, 250-256.

[15] Trefethen, L. N. Spectral Methods in MATLAB, SIAM, 2000. MR1776072 (2001c:65001)

[16] Vinogradova, O. I. Drainage of a thin liquid film confined between hydrophobic surfaces, Langmuir, 1995, 11, 2213-2220.

[17] Watanabe, K. and Akino, T., Drag reduction in laminar flow between two vertical coaxial cylinders, J. Fluids Eng., 1999, 121, 541-547.

[18] Watanabe, K., Takayama, T., Ogata, S. and Isozaki, S., Flow between two coaxial rotating cylinders with a high water-repellent wall, AIChE Journ., 2003, 49, 1956-1963.

[19] Weideman, J. A. C. and Reddy, S. C. A Matlab Differentiation Matrix Suite, ACM Trans. on Math. Software, 2000, 26 No. 4, 465-519. MR.1939962 (2003g:65004) 